International Journal of Engineering \& Technology, 7 (2.32) (2018) 473-476
International Journal of Engineering \& Technology
SPC
Website: www.sciencepubco.com/index.php/IJET
Research paper

\title{
Sentiment Analysis for Social Networks Using Machine Learning Techniques
}

\author{
Dorababu Sudarsa ${ }^{1}$, Siva kumar.P ${ }^{2}$, L.jagajeevan Rao $^{3}$ \\ ${ }^{1}$ Asst.Prof, KLE F , vijayawada, India . \\ ${ }^{2}$ Assoc. Professor KLE F, vijayawada, India, \\ ${ }^{3}$ Asst.Professor, $K L E F$, vijayawada, India. \\ *Corresponding author E-mail: dorababu.sudarsa@gmail.com
}

\begin{abstract}
The tremendous of the overall enormous net has conveyed a present day way of communicating the feelings of individuals. It's additionally a medium with a vast amount of data in which clients can see the assessment of different clients which can be ordered into exceptional entailment summons and are progressively more boom as a key component in decision making. This paper adds to the supposition assessment for customers assessment class that is utilized to analyze the records inside the type of the assortment of tweets wherein investigates are very unstructured and are both high fine or terrible, or somewhere in the middle of these . For this we first preprepared the dataset, after that extract the adjective from the dataset that has a couple of significance this is alluded to as capacity vector, at that point decided on the component vector posting and from that point accomplished device examining based write calculations particularly navie bayes, most entropy and svm along the edge of the semantic introduction based absolutely based on word net which extracts synonyms and similarity for the content characteristic. In the end, we measured the performance of the classifier in terms of considering, precision and accuracy.
\end{abstract}

Keywords:Machine Learning, Semantic Orientation, Sentiment Analysis, Twitter.

\section{Introduction}

The age of internet has modified the manner humans explicit their perspectives. it's miles now executed via weblog posts, on line dialogue forums, product review web sites and so forth. people depend upon this person generated content to a terrific quantity. while a person needs to shop for a product, they will appearance up its evaluations online earlier than taking a choice. the amount of user generated content is simply too huge for a

ordinary consumer to research. so that you could automate this, numerous sentiment analysis strategies are used. Symbolic strategies or understanding base approach and machine mastering techniques are the 2 important strategies used in sentiment analysis. Expertise base approach requires a large database of predefined feelings and an green expertise illustration for figuring out sentiments. Machine learning approach uses a education set to expand a sentiment classifier that classifies sentiments. Given that a predefined database of entire emotions is not required for system learning method, it's far alternatively easier than knowledge base technique. on this paper, we use special device getting to know techniques for classifying tweets.

The forefront of this exploration paper covers the assessment of the substance at the web covering bunches of zones that are wrapping exponentially in numbers notwithstanding in volumes as destinations are devoted to specific kinds of stock and that they have practical experience in storing up clients' audits from different locales, for example, Amazon and numerous others. Considerably twitter is where in the tweets convey surveys, however looking to harvest the general comprehension of these unstructured records (audits) might be extremely time ingesting. those unstructured measurements (audits) on a particular site are seen through by the customers and subsequently growing a picture about the items or offerings and accordingly at long last delivering a beyond any doubt judgment. these surveys are then being summed up to secure criticisms for unprecedented purposes to offer valuable audits in which we utilize assessment examination. Slant assessment is a procedure in which the dataset incorporates emotions, mentalities or evaluation which mulls over the way a human thinks [1]. In a sentence, hoping to secure the positive and the negative segment is a totally hard endeavor. the capacities used to classifications the sentences ought to have a totally Sturdy modifier with the expectation to abridge the assessment. Those $\mathrm{s}$ are even composed in unmistakable strategies which are not without issues concluded with the guide of the clients or the organizations making it extreme to group them. Opinion assessment influences clients to classes whether the information around the item is decent or no longer before they gather it. Advertisers and organizations utilize this investigation to catch about their administrations or items in the kind of way that it might be furnished as with regards to the individual's needs. There are two styles of machine picking up information of techniques which may be typically utilized for notion investigation, one is unsupervised and the other is managed [2]. Unsupervised becoming more acquainted with does not comprise of a class and that they don't give the ideal destinations at all and therefore conduct bunching. Managed learning is construct absolutely with respect to named dataset and hence the names are outfitted to the model all through the strategy. those sorted dataset are 
prepared to supply reasonable yields when experienced all through basic leadership. to help us to secure the opinion examination in a superior way, this exploration paper is based at the supervised system learning.

\section{Related Work}

There are basic techniques to unearth notions from content. They represents methodologies and contraption becoming acquainted with systems [2]. The accompanying two areas adapt to these methodologies.

A. Representative Techniques Much of the examination in unsupervised conclusion characterization utilizing emblematic procedures makes utilization of accessible lexical assets. Turney [3] utilized pack of-words approach for assessment examination. In that approach, connections between the individual words are not considered and a report is spoken to as a minor gathering of words. To decide the those qualities are joined with some total capacities.

He found the extremity of an audit in view of the normal semantic introduction of tuples separated from the survey where tuples are phrases having descriptors or verb modifiers. He found the semantic introduction of tuples utilizing the internet searcher Altavista. Kamps et al. [4] utilized the lexical database WordNet [5] to decide the passionate substance of a word along various measurements. They built up a separation metric on WordNet and decided the semantic introduction of descriptors. WordNet database comprises of words associated by equivalent word relations. Baroni et al. [6] built up a framework utilizing word space show formalism that beats the trouble in lexical substitution assignment. It speaks to the nearby setting of a word alongside its general dissemination. Balahur et al. [7] presented EmotiNet, an applied portrayal of content that stores the structure and the semantics of genuine occasions for a particular space. Emotinet utilized the idea of Finite State Automata to distinguish the passionate reactions activated by activities. One of the members of SemEval 2007 Task No. 14 [8] utilized coarse grained and fine grained ways to deal with distinguish assessments in news features. In coarse grained approach, they performed double characterization of feelings and in fine grained ap proach they grouped feelings into various levels. Knowledge base approach is found to be difficult due to the requirement of a huge lexical database. Since social network generates huge amount of data every second, sometimes larger than the size of available lexical database, sentiment analysis became tedious and erroneous.

B. Machine learning methods: gadget acing strategies utilize an instruction set and a test set for order. Tutoring set contains input work vectors and their comparing class names. utilizing this preparation set,a type display is developed which endeavors to order the information highlight vectors into comparing class names. at that point a test set is utilized to approve the adaptation by foreseeing the class names of inconspicuous element vectors. various gadget picking up learning of methodologies like credulous bayes (nb), most extreme entropy (me), and bolster vector machines (svm) are utilized to arrange feelings [9]. a portion of the abilities that can be utilized for slant write are day and age nearness, day and age recurrence, nullification, n-grams and grammatical feature [1].those capacities can be utilized to find the semantic introduction of words, expressions, sentences and that of records. Semantic introduction is the extremity which can be either positive or negative. dominos et al. [10] found that guileless bayes works legitimately for specific issues with very settled capacities. that is shocking as the central supposition of innocent bayes is that the capacities are fair. zhenniu et al. [11] presented a fresh out of the box new model wherein effective techniques are utilized for work determination, weight calculation and class. the new form is construct absolutely with respect to bayesian arrangement of tenets. ideal here weights of the classifier are balanced by method for utilizing advisor trade- mark and specific capacity. 'advisor highlight' is the records that speaks to a class and 'specific element' is the information that encourages in recognizing directions. the utilization of the ones weights, they figured the likelihood of every class and henceforth ventured forward the bayesian arrangement of rules. barbosa et al. [12] outlined a 2-step programmed opinion assessment method for arranging tweets. they utilized a boisterous tutoring set to lessen the marking endeavor in developing classifiers. above all else, they sorted tweets into subjective and objective tweets. from that point forward, subjective tweets are named as gigantic and negative tweets. celikyilmaz et al. [13] propelled an elocution principally based expression grouping approach for normalizing boisterous tweets. in elocution based word grouping, phrases having similar articulation are bunched and doled out typical tokens. they likewise utilized content handling systems like doling out comparable tokens for numbers, html joins, client identifiers, and objective business undertaking names for standardization. subsequent to doing standardization, they utilized probabilistic models to distinguish extremity vocabularies. they performed class the utilization of the boostexter classifier with these extremity dictionaries as capacities and obtained a decreased bungles cost. wu et al. [14] proposed an affect opportunity show for twitter assessment. in the event that @username is situated inside the casing of a tweet, it's far impacting development and it adds to affecting Shot. any tweet that begins with@username is a rewet that speaks to a motivated movement and it adds to empowered probability. they found that there is a solid relationship among these probabilities. pak et al. [15] made a twitter corpus through mechanically storing up tweets the utilization of twitter programming interface and routinely commenting on the ones the use of emotions. utilizing that corpus, they built an estimation classifier fundamentally based at the multinomial guileless bayes classifier that utilizations n-gram and poslabels as abilities. in that

Approach, there is a danger of mistake for the reason that feelings of tweets in preparing set are arranged exclusively basically based at the extremity of emotions. the instruction set is in like manner less proficient since it contains most straightforward tweets having emotions.

\section{Our Approach}

In our technique we used the twitter dataset and analyzed it.

This investigations sorted datasets the utilization of the unigram include extraction system. we utilized the structure wherein the preprocessor is done to the uncooked sentences which make it additional proper to perceive. so also, the unique gadget acing systems prepares the dataset with trademark vectors and after that the semantic assessment offers an extensive arrangement of equivalent words and similitude which bears the extremity of the substance. The entire depiction of the system has been portrayed in next sub segments and the piece outline of the same is graphically spoken to in fig.1. Chart of the strategy to issue a. prepreparing of the datasets the tweets contain various studies around the information which are communicated in particular strategies through people

The twitters dataset utilized on this work is now arranged. Arranged dataset has a negative and top notch extremity and therefore the examination of the data ends up smooth. the crude information having extremity is very inclined to irregularity and repetition. the charming of the certainties impacts the outcomes and subsequently which will enhance the pleasant, the crude insights is pre-handled. it offers with the planning that kills the rehashed words and accentuations and enhances the productivity the realities. for instance, "that artistic creation is beauuuutifull \#" in the wake of preprocessing proselytes to "painting staggering." further,"@geet is currently persevering" believers to "geet now dedi- 
cated". b. trademark extraction the ventured forward dataset after pre-handling has heaps of one of a kind homes. the capacity extraction approach, extricates the component (descriptive word) from the dataset. later this descriptor is utilized to demonstrate the high caliber and horrendous extremity in a sentence which is valuable for deciding the feeling of the general population the use of unigram show [15]. unigram show separates the modifier and isolates it. it disposes of the past and progressive word happening with the descriptive word in the sentences. for above case, i.e. "depict shocking" by means of unigram demonstrate, easiest delightful is removed from the sentence.

After the tutoring and classification we utilized semantic investigation. semantic investigation is gotten from the word net database in which each day and age is related with each extraordinary. This database is of English words that are connected together. on the off chance that expressions are close to each other, they're semantically comparable. additional particularly, we're ready to decide equivalent word like likeness. we delineate and take a gander at their relationship in the cosmology. the key mission is to apply the spared documents that contain terms and afterward investigate the comparability with the expressions that the individual employments of their sentences. in this manner it's miles gainful to uncover the extremity of the assessment for the clients. as an example inside the sentence's am fulfilled" the word "fulfilled" being a descriptor gets chose and is in correlation with the spared work vector for equivalent words. Give us a chance to expect 2 phrases; 'fulfilled' and 'happy' have a tendency to be particularly similar to the expression 'happy'. presently after the semantic assessment, 'happy' replaces 'fulfilled' which gives a fine extremity.

\section{Usage and End Result}

we utilized python and normal dialect gadget unit to teach and group the credulous bayes, most entropy and guide vector framework. by and large we utilized records set of length 19340 out of which 18340 have been utilized for preparing and 1000 for looking at. for training fig 2. show the overall waft of techniques.

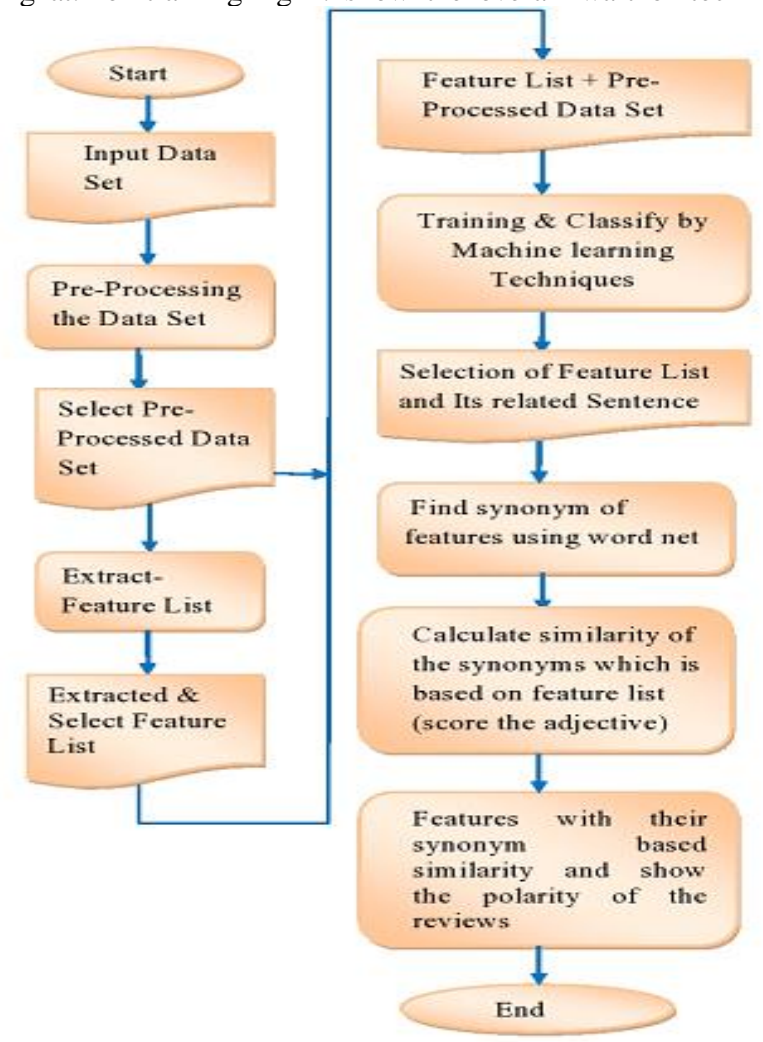

Fig. 2. Flow Diagram of the proposed methodology
The description of the manner in pseudo code shape is shown.

input: categorized dataset

output: fantastic and bad polarity with synonym of

words and similarity between phrases

step-1 pre-processing the tweets:

pre-processing ()

eliminate url:

eliminate unique symbols

convert to lower:

step-2 get the feature vector listing:

for $w$ in phrases:

update or more words

strip:

if (w in stop words)

hold

else:

append the document

go back function vector

step-3 extract features from feature vector listing:

for phrase in feature listing

capabilities=phrase in tweets words

go back features

step-4 integrate pre-processing dataset and feature vector listing

pre-processed record=course call of the record

stopwords=file direction name

function vector listing=document path of characteristic vector

listing

step-5 training the step 4

practice classifiers classes

step-6 discover synonym and similarity of the characteristic vec-

tor

for each sentences in function listing

extract feature vector within the tweets ()

for every function vector: $x$

for each function vector: $\mathrm{y}$

locate the $\operatorname{similarity}(\mathrm{x}, \mathrm{y})$

if (similarity>threshold)

fit observed

feature vector: $\mathrm{x}=$ feature vector: $\mathrm{y}$

classify $(\mathrm{x}, \mathrm{y})$

print: sentiment polarity with similar feature phrases

Fig. 3. Pseudo code of the procedure:

\section{Conclusion}

In this paper, we proposed a set of techniques of system gaining knowledge of with semantic evaluation for classifying the sentence and product opinions based totally on twitter facts. the important thing aim is to analyze a massive amount of reviews through using twitter dataset which are already classified. the naïve byes approach which offers us a better end result than the maximum entropy and svm is being subjected to unigram version which gives a better end result.

Than the use of it alone. Further the accuracy is once more progressed while the semantic evaluation word net is followed up by way of the above method taking it to $89.9 \%$ from $88.2 \%$. the training facts set can be improved to improve the feature vector related sentence identity process and can also expand word net for the summarization of the critiques. it may supply better visualization of the content in better manner with a view to be beneficial for the customers.

There are distinct symbolic and device gaining knowledge of strategies to become aware of sentiments from textual content. device gaining knowledge of techniques are less complicated and green than symbolic techniques. these techniques may be applied for twit- 
ter sentiment evaluation. there are sure issues at the same time as managing figuring out emotional key-word from tweets having multiple key phrases. it's miles additionally difficult to address misspellings and slang words. to deal with those issues, an efficient function vector is created through doing characteristic extraction in two steps after right preprocessing. In step one, twitter precise functions are extracted and delivered to the feature vector. after that, these features are removed from tweets and once more characteristic extraction is executed as if it's far accomplished on regular textual content. these features are also introduced to the characteristic vector. category accuracy of the function vector is examined the use of different classifiers like nave bayes, svm, maximum entropy and ensemble classifiers. most of these classifiers have almost similar accuracy for the new function vector. this feature vector performs nicely for digital products domain

\section{Conclusion}

In this paper we contributed a methodical survey of supposition examination and sentiment mining. The multifaceted nature of data Presentation and dimensionality, distinctive use necessities, the conclusion examination or sentiment mining developed as basic research objective thinking about that 10 years. This assess investigated the notion assessment method, contemporary assessment of The machine acing based absolutely assumption assessment designs found in late writing, effect of contraption learning .Conclusion investigation and plausible and limit thinks about focuses for predetermination look into. At some point or another, we finish up the Manuscript by utilizing saying that all the opinion assessment obligations are hard, because of the reality ability and know-how of the inconvenience and its answers are as yet obliged. The principle object is that it's far a home grown dialect handling undertaking, and Herbal dialect preparing has no simple issues. Be that as it may, numerous huge advances were made. Obvious to finish that the sentiment evaluation is having potential scope for destiny research and certainly one of that is exposing The scope of evolutionary computational or soft computing strategies and the hybridizing these techniques in the direction of Function extraction, selection to categories the sentiment.

\section{References}

[1] R. Feldman, "Techniques and Applications for Sentiment Analysis ," Communications of the ACM, Vol. 56 No. 4, pp. 82-89, 2013.

[2] Y. Singh, P. K. Bhatia, and O.P. Sangwan, "A Review of Stud-ies on Machine Learning Techniques," International Journal of Computer Science and Security, Volume (1) : Issue (1), pp. 70-84, 2007.

[3] P.D. Turney," Thumbs Up or Thumbs Down? Semantic Orientation Applied to Unsupervised Classification of Reviews," Proceedings of the 40th Annual Meeting of the Association for Computational Lin-guistics (ACL), Philadelphia, pp. 417-424, July 2002.

[4] Ch.L.Liu, W.H. Hsaio, C.H. Lee, and G.C.Lu, and E. Jou," Movie Rating and Review Summarization in Mobile Environment," IEEE Transactionson Systems, Man, and Cybernetics, Part C 42(3):pp.397-407, 2012. [5

[5] R.Liu,R.Xiong, and L.Song, "A Sentiment Classification Meth-od for Chinese Document," Processed of the 5th International Conference on Computer Science and Education (ICCSE), pp. 918 $922,2010$.

[6] A.khan,B.Baharudin, "Sentiment Classification Using Sentencelevel Semantic Orientation of Opinion Terms from Blogs," Processed on National Postgraduate Conference (NPC), pp. 1 - 7, 2011.

[7] L.Ramachandran,E.F.Gehringer, "Automated Assessment of Review Quality Using Latent Semantic Analysis," ICALT, IEEE Computer Society, pp. 136-138, 2011.

[8] B. Pang and L. Lee, Opinion mining and sentiment analysis, Foundations and Trends in Information Retrieval, vol. 2, pp. 8-10, 2008.

[9] B. G. Malkiel, A Random Walk Down Wall Street: Including a LifeCycle Guide to Personal Investing. WW Norton \& Company, 1999.[3

[10] J. Bollen, H. Mao and X. Zeng, Twitter mood predicts the stock market, Journal of Computational Science, vol. 2, pp. 1-8, 2011.
[11] M. A. Russell, Mining the Social Web: Data Mining Facebook, Twitter, LinkedIn, Google+, GitHub, and More. O'Reilly Media, Inc, 2013.

[12] L. Bing, K. C. Chan and C. Ou, Public sentiment analysis in twitter data for prediction of a company's stock price movements, in EBusiness Engineering (ICEBE), 2014 IEEE 11th International Conference on, 2014, pp. 232-239.

[13] M. Mittermayer, Forecasting intraday stock price trends with text mining techniques, in System Sciences, 2004. Proceedings of the 37th Annual Hawaii International Conference on, 2004, pp. 10-pp.

[14] R. Socher, A. Perelygin, J. Y. Wu, J. Chuang, C. D. Manning, A. Y.Ng and C. Potts, Recursive deep models for semantic compositionality over a sentiment treebank, in Proceedings of the Conference on Empirical Methods in Natural Language Processing (EMNLP), 2013, pp. 1642.

[15] M. Sokolova and G. Lapalme, A systematic analysis of performance measures for classification tasks, Information Processing \& Management, vol. 45, pp. 427-437, 2009.

[16] Go, R. Bhayani and L. Huang, Twitter sentiment classifi-cation using distant supervision, CS224N Project Report, Stan-ford, pp. 112,2009 .

[17] Bermingham and A. F. Smeaton, Classifying sentiment in microblogs: Is brevity an advantage? in Proceedings of the 19th ACM International Conference on Information and Knowledge Management, 2010, pp. 1833-1836.

[18] Pak and P. Paroubek, Twitter as a corpus for sentiment analysis and opinion mining. in Lrec, 2010, pp. 1320-1326.

[19] L. Barbosa and J. Feng, Robust sentiment detection on twitter from biased and noisy data, in Proceedings of the 23rd Interna-tional Conference on Computational Linguistics: Posters, 2010, pp. 36-44.

[20] Oh and O. Sheng, Investigating predictive power of stock micro blog sentiment in forecasting future stock price directional movement, 2011.

[21] Tayal and S. Komaragiri, Comparative Analysis of the Impact of Blogging and Micro-blogging on Market Performance, International Journal, vol. 1, pp. 176-182, 2009.

[22] M. Sokolova and G. Lapalme, A systematic analysis of performance measures for classification tasks, Information Processing \& Management, vol. 45, pp. 427-437, 2009.

[23] S. Dreiseitl and L. Ohno-Machado, Logistic regression and artificial neural network classification models: a methodology re-view, J.Biomed. Inform., vol. 35, pp. 352-359, 2002.

[24] Gartner, http://www.gartner.com/newsroom/id/766215 ac-cessed on Mar 30, 2015.

[25] IDC, http://blogs.idc.com/ie/?p=190, accessed on Mar 30,2 015.

[26] The451group,www.451group.com/reports/execu=ve_summary.php ?id=619, accessed on Mar 30, 2015. 